\title{
Lymphödem
}

\section{Praxis-EDV hilft bei der Heilmittel-Verordnung}

\author{
Für Ärzte, die Patienten mit Lymphödem behandeln, hat es 2017 \\ gleich mehrere Neuerungen gegeben. Sie betreffen die Kodierung \\ der Diagnosen und die Erleichterung der Heilmittel-Verordnung. \\ Hilfen erhalten Ärzte via Praxis-EDV, eine neue Leitlinie gibt inhaltliche \\ Orientierung.
}

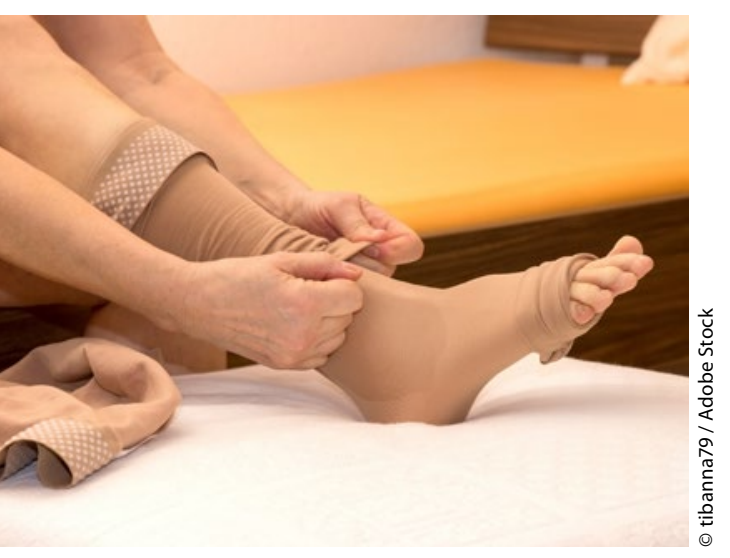

D en Schlusspunkt setzte Ende Mai die Veröffentlichung des Beschlusses des Gemeinsamen Bundesausschusses (G-BA) im Bundesanzeiger. Damit wurde der G-BA-Beschluss zur Heilmittelrichtlinie mit Bezug auf Patienten mit Lymphödemen in Kraft gesetzt. Infolgedessen sind jetzt auch Lymphödeme des Stadiums II als Erkrankungen mit langfristigem Heilmittelbedarf anerkannt. In der Folge unterliegen Verordnungen manueller Lymphdrainage für Lymphödeme des Stadiums II und III nicht der Wirtschaftlichkeitsprüfung und können auch langfristig verordnet werden.

\section{Ärzte sollen fünfstellig kodieren}

Der G-BA hatte mit seinem Beschluss auf die Änderungen in der ICD-10GM-2017 reagiert, die es nun erlauben, statt des vorher meist genutzten undiffe- renzierten Diagnoseschlüssels $\quad$ I89.0 fünfstellige Codes zur Differenzierung nach Stadien des Lymphödems zu nutzen. So steht der Schlüssel I89.00 für ein Lymphödem der oberen und unteren Extremitäten im Stadium I, I89.01 für Stadium II und I89.02 für ein Lymphödem am selben Ort im Stadium III.

Eine gute inhaltliche Ergänzung für Ärzte, die Patienten mit Lymphödemen betreuen, bietet die aktualisierte S2kLeitlinie der Arbeitsgemeinschaft der Wissenschaftlich-Medizinischen Fachgesellschaften (AWMF), die seit Ende Mai verfügbar ist. In der Leitlinie ist unter anderem aufgeführt, wie ein Arzt nach den verschiedenen Stadien der Lymphödeme differenzieren kann (Tab. 1). Denn ohne die genaue Feststellung des Stadiums können Ärzte natürlich auch nicht die entsprechenden neuen fünfstelligen ICD-10-Schlüssel dokumentieren, die in der Liste aufgeführt sind, für die der langfristige Heilmittelbedarf - also die manuelle Lymphdrainage (MLD) - anerkannt ist.

\section{Software erkennt ICD-10-Codes}

Die entsprechenden Diagnoseschlüssel für langfristigen Heilmittelbedarf (Anlage 2 der Heilmittelrichtlinie) sind seit April in der Software hinterlegt. Damit sollte die Praxissoftware bei Verordnung über das Muster 13 automatisch über den verwendeten ICD-10-Schlüssel erkennen, ob die Heilmittelverordnung unter die Wirtschaftlichkeitsprüfung fällt oder ob die Verordnung aufgrund der Schwere der Erkrankung und aufgrund ihrer zu erwartenden Dauer nicht budgetrelevant ist und also langfristig verordnet werden kann.

Der Ende Mai in Kraft getretene Beschluss des G-BA für Patienten mit Lymphödemen soll über die erweiterte Anlage 2 mit den entsprechenden ICD10-Diagnosen seit Anfang Juli in den Programmen hinterlegt sein.

Die Umsetzung der geänderten Heilmittelrichtlinie war von der KBV bis zum 1. April verlängert worden, sollte aber inzwischen in allen Programmen realisiert sein. Die Erweiterung der Liste dürfte für die Softwarehersteller kein Problem mehr sein. Wie eine Praxissoftware anzeigt, ob eine Heilmittelverordnung ins Budget fällt oder nicht, hat jedes Unternehmen für sein(e) Programm(e) umgesetzt. Die Neuerungen tragen jedenfalls dazu bei, dass Ärzte die Verordnung von Heilmitteln wesentlich besser steuern können als vor dem 1. April.

Hauke Gerlof

Tab. 1: Stadien eines Lymphödems
$\begin{array}{ll}\text { Stadium 0 } & \begin{array}{l}\text { subklinisches Stadium: kein } \\ \text { klinisch apparentes Lymph- } \\ \text { ödem, teils pathologisches } \\ \text { Lymphszintigramm }\end{array} \\ \text { Stadium I } & \begin{array}{l}\text { Ödem von weicher } \\ \text { Konsistenz; Hochlagern } \\ \text { reduziert die Schwellung }\end{array} \\ \text { Stadium II } & \begin{array}{l}\text { Ödem mit sekundären } \\ \text { Gewebeveränderungen; }\end{array} \\ & \begin{array}{l}\text { Hochlagern beseitigt die } \\ \text { Schwellung nicht } \\ \text { deformierende harte }\end{array} \\ \text { Stadium III } & \begin{array}{l}\text { Schwellung, zum Teil lobäre } \\ \text { Form mit teils typischen } \\ \text { Hautveränderungen }\end{array} \\ \text { Quelle: S2k-Leitlinie der AWMF „Diagnostik und }\end{array}$
Therapie der Lymphödeme“, Reg.-Nr. 058-001

